\title{
Surgical Start Time Impact on Hospital Length of Stay for Elective Inpatient Procedures
}

Christopher P. Cifarelli ${ }^{1,2}$, John P. McMichael ${ }^{3}$, Alex G. Forman ${ }^{4}$, Paul A. Mihm ${ }^{5}$, Daniel T. Cifarelli ${ }^{6}$, Mark R. Lee ${ }^{6}$, Wallis Marsh ${ }^{7}$

1. Neurological Surgery, West Virginia University School of Medicine, Morgantown, USA 2. Radiation Oncology, West Virginia University School of Medicine, Morgantown, USA 3. Information Technology, Cleveland Clinic, Cleveland, USA 4. Strategic Analytics, West Virginia University School of Medicine, Morgantown, USA 5. Surgical Services, West Virginia University School of Medicine, Morgantown, USA 6. Neurosurgery, West Virginia University School of Medicine, Morgantown, USA 7. Surgery, West Virginia University School of Medicine, Morgantown, USA

Corresponding author: Christopher P. Cifarelli , cpcifarelli@gmail.com

\section{Abstract \\ Background}

Hospital length of stay (LOS) remains an important, albeit nonspecific, metric in the analysis of surgical services. Modifiable factors to reduce LOS are few in number and the ability to practically take action is limited. Surgical scheduling of elective cases remains an important task in optimizing workflow and may impact the post-surgical LOS.

\section{Methods}

Retrospective data from a single tertiary care academic institution were analyzed from elective adult surgical cases performed from 2017 through 2019. Emergent or urgent add-on cases were excluded. Variables included primary procedure, age, diabetes status, American Society of Anesthesiologists (ASA) class, and surgical start time. Analysis of the median LOS following surgery was performed using Mann-Whitney tests and Cox hazards model. Matched-cohort analysis of mean total hospitalization costs was performed using the Student's t-test.

\section{Results}

9,258 patients were analyzed across five surgical service lines, of which 777 patients had surgical start time after 3 PM. The median LOS for the after 3 PM group was 1 day longer than the before 3 PM start time cohort ( 3.0 vs $2.1, \mathrm{p}<0.001$ ). Service line analysis revealed increased LOS for Orthopedics and Neurosurgery (3.0 vs $1.9, \mathrm{p}<0.001 ; 3.0$ vs $2.0, \mathrm{p}<0.05$ ). Multivariate analysis confirmed that start time before 3 PM predicted shorter LOS $(H R=1.214,1.126-1.309 ; \mathrm{p}<0.001)$. Case-matched cost analysis for frequently performed orthopedic and neurosurgical cases with an after $3 \mathrm{PM}$ start time failed to demonstrate a significant difference in total hospital charges.

Review began 06/08/2021 Review ended 06/29/2021 Published 07/08/2021

\section{๑) Copyright 2021}

Cifarelli et al. This is an open access article distributed under the terms of the Creative Commons Attribution License CC-BY 4.0., which permits unrestricted use, distribution, and reproduction in any medium, provided the original author and source are credited.

\section{Conclusion}

Optimization of surgical services scheduling to increase the proportion of elective surgical cases started before 3 PM has the potential to decrease post-surgical LOS for adult patients undergoing Orthopedic or Neurosurgical procedures.

Categories: Quality Improvement

Keywords: length of stay, elective surgery, surgery start time, after-hours care, surgical scheduling

\section{Introduction}

The operational efficiency of elective surgical procedures with planned hospital admissions is dependent on multitude of factors, only a small portion of which are modifiable. Patient age, comorbid states, surgical experience of the operative staff, and the underlying diagnosis are all areas in which the patients and providers have limited control. Conversely, parameters such as the choice of peri-operative medications, post-operative mobilization plans and surgical start time are slightly more adaptable and offer the potential opportunity for optimization.

Hospital length of stay (LOS) remains one of the most studied endpoints in outcomes research as it relates to efficiency in care delivery. Many studies have focused on the use of opioids, glycemic levels, and cardiac function in the development of predictive models, usually as they relate to specific surgical procedures [1]. From a resource utilization standpoint, LOS variation impacts the ability to offer elective and nonelective services to additional patients, while also fiscally creating burdens for services with fixed reimbursement schedules, regardless of the numbers of hospitalization days [2]. 
Attempts to transition appropriate elective surgical procedures from hospital admission status to same day surgery have been moderately successful from the perspectives of both patients and healthcare delivery systems [3-5]. Unfortunately, a significant proportion of elective surgeries, especially those involving orthopedic or cancer care, still require some form of inpatient hospitalization. Recent stresses in the U.S. healthcare system as related to the COVID-19 pandemic have exacerbated the need for timely hospital discharge for elective surgical patients based on system throughput and exposure risk. As many healthcare systems look to expand the use of non-traditional operative days (weekends) and times of day (after 3 PM start times) to compensate for access limitations early in the pandemic, there is an unknown in the impact that such modifications will have to surgical outcomes, specifically hospital LOS.

The current study reviews the single-institutional elective surgical experience of a large academic medical center that serves a rural patient population spread over approximately 25,000 square miles for a three-year period between 2017 and 2019 with analysis of hospital LOS as a function surgical start time. These data have been previously discussed in preprint format (DOI: 10.21203/rs.3.rs-94936/v1) prior to peer-review publication.

\section{Materials And Methods}

Data inquiries were made of the central surgical scheduling database and hospital charge master dataset for elective adult surgical cases performed at West Virginia University Ruby Memorial Hospital from 2017 through 2019. WVU IRB exemption was granted non-human-subject research. De-identified data were coded based on age at the time of surgery, ASA class, diabetes status, surgical start time based on anesthesia release, length of post-surgical hospital stay and type of surgical service performing the procedure. Cases involving patients under 18 years of age, emergency/urgent non-elective status, post-surgical hospitalizations greater than 90 days, planned same-day surgeries, or weekend elective cases were excluded from the current analysis. In addition, patients who expired during hospitalization for an elective procedure were not included in the data set. Surgical start times were dichotomized as pre- and post-3 PM based on the institutional pattern of standard daytime change of shift that affects more than $75 \%$ of operating room personnel.

Univariate analysis of the effect of surgical start time was performed using the non-parametric MannWhitney test based on the non-normal distribution of post-surgical hospital length of stay data. Multivariate analyses were performed according to the Cox proportional hazard model using variables of age $(<65$ years), diabetic status, American Society of Anesthesiologists (ASA) classification, and surgical start time. Proportional hazard modeling was utilized based on the potential of any of the selected parameters to impact the length of hospitalization and time to event was defined as admission length of stay from operating room discharge to facility discharge. Case matching for total hospitalization charge analysis was performed based on primary procedural CPT code, patient age at the time of surgery, and gender. Cost analysis was based on charges only, not absolute costs or reimbursement, and performed on cases where the patient was admitted for hospitalization on the day of the procedure. In order to eliminate the existence of pre-surgical hospital charges during the admission, only cases admitted on the day of surgery were included. All statistical analyses were performed using Statistical Package for the Social Sciences (SPSS) V.26 (IBM Corp, Armonk, NY) with significance at $\mathrm{p}<0.05$.

\section{Results}

\section{Patient demographics}

Between 2017 and 2019, a total of 9,258 adult elective surgical cases were performed amongst five primary surgical service lines, including General Surgery, Surgical Oncology, Orthopedic Surgery, Cardiac Surgery, and Neurological Surgery (summarized in Table 1). 


\section{Cureus}

\begin{tabular}{|c|c|c|c|c|c|c|c|}
\hline & \multirow{2}{*}{ Total cases } & Start time & Start time & \multirow{2}{*}{ Mean age ( \pm SEM) } & \multirow{2}{*}{ Median age } & \multirow{2}{*}{$\begin{array}{l}\text { Diabetic patients } \\
\text { (\% of total) }\end{array}$} & \multirow{2}{*}{$\begin{array}{l}\text { ASA classes } \\
\text { (\% of total) }\end{array}$} \\
\hline & & Before 3 PM & After 3 PM & & & & \\
\hline \multirow{4}{*}{ General Surgery } & \multirow{4}{*}{1723} & \multirow{4}{*}{1546} & \multirow{4}{*}{177} & \multirow{4}{*}{$49.8 \pm 0.373$} & \multirow{4}{*}{50} & \multirow{4}{*}{$411(23)$} & $1: 7(0.4)$ \\
\hline & & & & & & & 11: $1 / 8(10)$ \\
\hline & & & & & & & III: 1271 (75) \\
\hline & & & & & & & IV: 224 (13) \\
\hline \multirow{4}{*}{ Orthopedic Surgery } & \multirow{4}{*}{4335} & \multirow{4}{*}{4061} & \multirow{4}{*}{274} & \multirow{4}{*}{$59.9 \pm 0.220$} & \multirow{4}{*}{62} & \multirow{4}{*}{$726(17)$} & I: 63 (1.5) \\
\hline & & & & & & & II: 1010 (24) \\
\hline & & & & & & & III: 2952 (70) \\
\hline & & & & & & & IV: 206 (5) \\
\hline \multirow{4}{*}{ Surgical Oncology } & \multirow{4}{*}{1261} & \multirow{4}{*}{1163} & \multirow{4}{*}{98} & \multirow{4}{*}{$58.8 \pm 0.418$} & \multirow{4}{*}{60} & \multirow{4}{*}{$204(16)$} & I: $5(0.4)$ \\
\hline & & & & & & & II: 206 (17) \\
\hline & & & & & & & III: 937 (76) \\
\hline & & & & & & & IV: 92 (8) \\
\hline \multirow{4}{*}{ Neurological Surgery } & \multirow{4}{*}{1640} & \multirow{4}{*}{1427} & \multirow{4}{*}{213} & \multirow{4}{*}{$55.5 \pm 0.375$} & \multirow{4}{*}{57} & & I: $10(0.6)$ \\
\hline & & & & & & & II: 292 (18) \\
\hline & & & & & & 200 (10) & III: 1182 (73) \\
\hline & & & & & & & IV: 137 (9) \\
\hline & & & & & & & I: $0(0)$ \\
\hline & & & & & & & II: 5 (2) \\
\hline Caralac surgery & 299 & 284 & 15 & $58.8 \pm 0.862$ & 61 & /2(24) & III: 67 (27) \\
\hline & & & & & & & IV: 178 (71) \\
\hline & & & & & & & I: 85 (1) \\
\hline & & & & & & & II: 1691 (19) \\
\hline Iotal & 9258 & 8481 & 777 & $57.1 \pm 0.159$ & 59 & 1681 (18) & III: 6409 (71) \\
\hline & & & & & & & IV: 837 (9) \\
\hline
\end{tabular}

\section{TABLE 1: Case demographics by surgical service line.}

The total number of cases performed between 2017 through 2019 on adult patients (18 years and older) with a planned elective post-operative admission were stratified according to surgical start time, age, diabetes status, and American Society Anesthesiologist (ASA) classification.

The greatest proportion of cases were attributed to the Orthopedic Surgery service, with 4335 cases comprising $46 \%$ of the total cases analyzed. The median age was 59 years at the time of surgery, with 1681 patients (18\%) having an admission diagnosis of diabetes mellitus. The American Society of Anesthesiologists (ASA) Physical Status Classification System rating were applied to each patient at the time of admission, with over $70 \%$ of total patients receiving an ASA III class. This trend was preserved across all service lines, except Cardiac Surgery, where 71\% of patients were designated ASA IV. The surgical start time was coded according to cases starting before or after 3 PM, with 8,481 (92\%) of cases starting before 3 PM.

\section{Hospital length of stay based on surgical start time}

The percentage of cases with start time before 3 PM ranged from $87 \%$ to $95 \%$ according to service line, with Orthopedic and Cardiac Surgery preserving the highest percent of total cases with before 3 PM commencement (94\% and 95\%, respectively) (Table 2). 


\section{Cureus}

\begin{tabular}{|c|c|c|c|c|c|}
\hline & \multirow{3}{*}{ Before 3 PM start time (\%) } & \multirow{3}{*}{ After 3 PM start time (\%) } & Median LOS & Mean LOS & \multirow{3}{*}{$\mathbf{p}$} \\
\hline & & & Before 3 PM & Before 3 PM & \\
\hline & & & After 3 PM & After 3 PM & \\
\hline \multirow{2}{*}{ General Surgery } & \multirow{2}{*}{$1546(88)$} & \multirow{2}{*}{$177(10)$} & 2.0 & 5.1 & \multirow{2}{*}{0.768} \\
\hline & & & 1.9 & 7.6 & \\
\hline \multirow{2}{*}{ Orthopedic Surgery } & \multirow{2}{*}{4061 (94) } & \multirow{2}{*}{$274(6)$} & 1.9 & 3.5 & \multirow{2}{*}{$<0.001$} \\
\hline & & & 3.0 & 6.3 & \\
\hline \multirow{2}{*}{ Surgical Oncology } & \multirow{2}{*}{$1163(92)$} & \multirow{2}{*}{$98(8)$} & 3.9 & 5.7 & \multirow{2}{*}{0.606} \\
\hline & & & 4.5 & 5.5 & \\
\hline \multirow{2}{*}{ Neurological Surgery } & \multirow{2}{*}{$1427(87)$} & \multirow{2}{*}{$213(13)$} & 2.0 & 4.6 & \multirow{2}{*}{$<0.05$} \\
\hline & & & 3.0 & 5.4 & \\
\hline \multirow{2}{*}{ Cardiac Surgery } & \multirow{2}{*}{$284(95)$} & \multirow{2}{*}{$15(5)$} & 6.1 & 9.9 & \multirow{2}{*}{0.324} \\
\hline & & & 5.7 & 10.3 & \\
\hline \multirow{2}{*}{ Total } & \multirow{2}{*}{8481 (92) } & \multirow{2}{*}{777 (8) } & 2.1 & 4.5 & \multirow{2}{*}{$<0.001$} \\
\hline & & & 3.0 & 6.3 & \\
\hline
\end{tabular}

\section{TABLE 2: Hospital length of stay (LOS) based on surgical start time.}

The mean and median LOS were compared for all cases and within each service line. Statistical differences in median LOS were determined by the Mann-Whitney $U$ test based on the non-normal distribution of LOS data with significance noted at $p<0.05$. Both Orthopedic and Neurological services demonstrated and an increase in median LOS of 1 day for cases started after 3 PM.

The median post-surgical length of stay for all cases was 2.1 days for the before 3 PM group and three days for the after 3 PM group ( $<$ 0.001). Mean values for these same groups remained shorter for the before 3 PM start time group compared to the after 3 PM group, although the non-normality of the LOS data precluded the ability to accurately perform a statistical test based on the means, with a skewness value of $4.78 \pm$ 0.025. Of the service lines examined, Orthopedic Surgery and Neurological Surgery were both found to have a significantly shorter median LOS in the before 3 PM start time group based on the Mann-Whitney Test $(\mathrm{U}=$ 413072, $\mathrm{p}<0.001 ; \mathrm{U}=139251, \mathrm{p}<0.05$, respectively).

\section{Predictors of post-surgical length of stay}

Semi-parametric multivariate analysis revealed predictors of post-operative length of stay according to the Cox proportional hazards model, including age ( $<65$ years), ASA class (I-IV), diabetes mellitus status, and surgical start time (before $3 \mathrm{PM}$ ) were used in the modeling. Hazard ratios (HR) demonstrated significance for shorter post-surgical length of stay based on age $<65$ (HR $=1.046,95 \% \mathrm{CI} 1.001-1.093)$, absence of a diabetes mellitus diagnosis ( $\mathrm{HR}=1.137,95 \% \mathrm{CI} 1.077-1.20)$, and surgical start time before $3 \mathrm{PM}$ ( $\mathrm{HR}=1.214$, 95\%CI 1.126-1.309) (Table 3). 


\section{Cureus}

\begin{tabular}{|c|c|c|c|c|c|}
\hline & & \multirow[b]{2}{*}{$H R \operatorname{Exp}(B)$} & \multicolumn{2}{|c|}{ 95\% Cl for $\operatorname{Exp}(\mathrm{B})$} & \multirow[b]{2}{*}{$\mathrm{p}$} \\
\hline & & & Lower & Upper & \\
\hline Age (<65 years) & & 1.046 & 1.001 & 1.093 & $<0.05$ \\
\hline \multirow[t]{4}{*}{ ASA } & 1 & 0.134 & 0.033 & 0.544 & 0.005 \\
\hline & 2 & 0.129 & 0.032 & 0.517 & $<0.005$ \\
\hline & 3 & 0.096 & 0.024 & 0.386 & 0.001 \\
\hline & 4 & 0.048 & 0.012 & 0.194 & $<0.001$ \\
\hline Non-diabetic & & 1.137 & 1.077 & 1.20 & $<0.001$ \\
\hline Start time before 3 PM & & 1.214 & 1.126 & 1.309 & $<0.001$ \\
\hline
\end{tabular}

TABLE 3: Cox proportion hazard model of predictors of LOS.

Age, ASA classification, diabetes status, and surgical start times were dichotomized and analyzed using Cox regression methodology. Hazard ratios $(\mathrm{HR}, \operatorname{Exp}(B))$ and the associated $95 \%$ confidence intervals $(\mathrm{Cl})$ were expressed with $\mathrm{p}<0.05$ in all categories. Positive predictors of a shorter LOS included age $<65$ years, non-diabetic status and surgical start time before 3 PM.

\section{Surgical case categories for orthopedic surgery \& neurological surgery}

The identification of increased LOS in patients with surgical start time after 3 PM in the Orthopedic and Neurological service lines prompted an evaluation of the case mixtures as defined by body location in Orthopedics and sub-specialty in Neurological surgery. Based on primary CPT codes, the majority of adult elective orthopedic surgery cases performed after 3 PM were leg/ankle cases (45\%) and evenly distributed among the other anatomical sites including pelvis/hip, femur/knee, arm/elbow, and spine, ranging from $8.2 \%$ to $13 \%$ (Table 4 ).

Orthopedic cases by start time

\section{Surgical site}

Pelvis/hip

Femur/knee

Leg/ankle

Foot

Shoulder/humerus

Arm/elbow

Hand

Spine

Neurosurgical cases by start time

Cranial

Spine

Peripheral/functional

\section{Before $3 \mathrm{PM} \mathrm{N}=$}

1406

1046

387

93

54

97

14

770

651

673

45
After 3 PM N= (\% of total after 3 PM)

$22(8.2 \%)$

$30(11.2 \%)$

122 (45.4\%)

7 (2.6\%)

8 (3.0\%)

35 (13.0\%)

1 (0.4\%)

32 (11.9\%)

98 (48.3\%)

99 (48.8\%)

$6(3.0 \%)$
749

772

\section{Total $\mathrm{N}=$}

1428

1076

509

100

62

132

15

802

51

\section{TABLE 4: Orthopedic and neurosurgical case categories.}

Orthopedic cases were organized based on anatomic region and stratified according to surgical start time. Neurosurgical cases were divided into cranial, spinal, and peripheral/functional cases and stratified by start time. 


\section{Total hospital charge analysis for orthopedic surgery \& neurological surgery}

The three highest frequency cases in Orthopedics were total hip arthroplasty (CPT 27130, n = 12), arthroplasty knee revision (CPT 27486; $\mathrm{n}=17$ ) and arthroplasty knee total (CPT 27447; $\mathrm{n}=14$ ). Within Neurological Surgery, the highest frequency cases were cranioplasty (CPT 62148; $\mathrm{n}=7$ ), craniotomy for tumor (CPT 61546; $\mathrm{n}=9$ ), and anterior cervical discectomy and fusion (CPT 22551; $\mathrm{n}=15$ ) (Table 5).

\begin{tabular}{|c|c|c|c|}
\hline CPT primary procedure & Before 3 PM start mean total charges & After 3 PM start mean total charges & $\mathbf{p}$ \\
\hline \multicolumn{4}{|l|}{ Orthopedic Surgery } \\
\hline 27130 Arthroplasty Total Hip & 48,306 & 53,880 & 0.49 \\
\hline 27486 Arthroplasty Knee Revision & 85,176 & 86,591 & 0.87 \\
\hline 27447 Arthroplasty Knee Total & 40,540 & 50,644 & 0.09 \\
\hline \multicolumn{4}{|l|}{ Neurological Surgery } \\
\hline 62148 Cranioplasty & 40,782 & 50,412 & 0.14 \\
\hline 61546 Craniotomy for Tumor & 109,212 & 85,729 & 0.14 \\
\hline 22551 Discectomy Spine Anterior Cervical with Fusion & 53,541 & 51,889 & 0.82 \\
\hline
\end{tabular}

\section{TABLE 5: Mean total hospital charge difference based on surgical start time.}

Cases without precedent hospitalization relative to the primary surgical procedure were matched between the before 3 PM and after 3 PM start time cohorts. The three most frequent procedures based on CPT code were compared for differences in mean total hospital charges. No statistically significant differences were noted between the two groups $(p>0.05)$.

No statistically significant differences were found ( $p>0.05$ ) among cases matched based on CPT code, age, or gender, nor were any consistent trends noted, with mean total charges being slightly higher in the after 3 PM group within Orthopedic Surgery and for the cranioplasty procedure within the Neurological Surgery case matched cohorts.

\section{Discussion}

Value propositions in healthcare remain at the forefront of both patient and provider interests. To that end, government agencies, private insurers, and patient advocacy groups have all spent considerable time and effort in attempting to define reliable metrics that are reflective of optimal value in care. Perhaps the most simplified measure of value for all involved in delivering and receiving healthcare service is time. Although lacking in specific information regarding outcome and/or satisfaction, hospital length of stay has been the predominant measure in outcomes research, largely based on the universal understanding that the reduction in hospitalization time is a positive attribute.

The impact of elective surgical procedures on overall operating costs for US hospitals was highlighted by the Healthcare Cost and Utilization Project published through the Agency for Healthcare Research and Quality (AHRQ) where only $29 \%$ of hospitalizations involved a surgical procedure, but these hospitalizations accounted for $48 \%$ of the $\$ 387$ billion in hospital costs in 2011 [6]. Of interest, this study also concluded that hospital admissions involving OR procedures were associated with a longer mean length of stay (5.0 vs 4.4 days) compared to admissions without surgery and the percent of elective surgical admissions was more than 3 -fold higher than non-surgical admissions ( $48 \%$ vs $13 \%$ of total $\mathrm{N}$ ). Overall, this work demonstrated the significant proportion of expenditure and resource utilization accompanying surgical admissions in the US, while alluding to the need to identify areas for cost reduction.

Elective surgical procedures present a number of opportunities for augmentation in the need for inpatient hospitalizations. Compared to urgent or emergent procedures, the elective surgeries allow for a controlled approach to post-surgical disposition planning and a reduction in patient/family anxiety. From a system occupancy perspective, the transition away from universal overnight hospitalization for short procedures to same-day surgery has been seen in a multitude of areas [7-9]. Yet, the ability to transition an overnight observation case to a same-day surgery case is not universal, regardless of the case complexity. In the setting of anterior cervical discectomy and fusion, a common spine procedure that has seen a trend toward same-day discharge, Mayo et al found that the time of day of the procedure was predictive of the ability to 
discharge same day. In that study, a "late” mean surgical start time of 12:19 (range: 9:10-17:51) resulted in a higher likelihood of requiring overnight hospitalization (HR 1.6 $\pm 0.30 ; p=0.010$ ) [10]. Of course, the limitations of same-day discharge in this situation is affected by mandatory minimum post-anesthetic observation time, unlike the cases in which overnight admission is guaranteed post-operatively.

In the current study, we identify a significant LOS effect among elective cases with planned hospital admissions in which surgical start time is documented after 3 PM, which accounts for $8.4 \%$ of all 9258 cases analyzed. Based on service line demarcation, only Orthopedic and Neurological surgery appeared to be effected, with these cases representing $65 \%$ of the total cases analyzed. Unfortunately, a more detailed analysis of these cases based on surgical site, failed to demonstrate a significant predominance of a particular procedure that could account for the time of day effect. In fact, the distribution in Neurosurgical cases between cranial and spine was precisely even at $48 \%$ of all after 3 PM cases within that service line, providing an argument against the impact of bias in case scheduling. While the multivariate analysis of age, ASA classification, and diabetes status all found significant predictors of shorter LOS in the non-diabetic, ASA Class I patient under the age of 65 , the start time before $3 \mathrm{PM}$ was the most powerful indicator (HR $1.214 ; \mathrm{p}<0.001)$.

As with most modern value assessments in healthcare, total hospital charges accrued during an episode of care remain an important metric. Continued evolution of the reimbursement process will likely increase the importance of total charges for all hospital systems, with potential likelihood that fixed payment schedules may eventually become universal based on diagnostic code [11]. Experiences with bundled payments in orthopedic and spinal procedures has resulted in mixed response with respect to total episode costs, without differences in length of stay [12]. Our analysis failed to demonstrate a significant difference in case-matched charges for procedures performed in the before or after 3 PM cohorts, although the trend toward higher charge accumulation followed with the increased LOS in the after 3 PM for four out of the six procedures analyzed.

The demarcation of the 3 PM start time was not arbitrary, but based on the inherent shift changes that impact operating room staff, including nursing, surgical technologists, and anesthetists. Given the mixture of room personnel, there was the highest likelihood that at least one team member transitions off shift and is replaced by another that was not present for the start of the case. In addition, previous literature has supported the concept that adverse anesthetic effects are more likely to occur in patients with surgical start time after 3 PM [13]. Similarly, anesthetic handoffs are more likely during evening or weekend cases in a large series analysis of the ACS NSQIP, where documented post-operative complications are more likely, although no causal relationship has been established, nor is the presence of a handoff predictive of a postsurgical morbidity after correcting for the co-variate of time of day [14].

Our data is contrasted to that of many other studies that examine the time of day effect on procedural safety, namely in the overall survival or development of co-morbidities, such as infection, cardiac event, or stroke [15-18]. With respect to morbidities, the literature is dichotomous with some studies dismissing the impact of surgical start time, while others underscoring the importance of such. Of interest, a clear time of day effect has been described in serval cardiac surgery studies, noting higher blood transfusion rates and even increased mortality $[19,20]$. With this in mind, our institution's avoidance of late starts in the Cardiac service line ( $5 \%$ of total cases) in understandable.

The limitations of this work include its single institutional, retrospective nature and the lack of outcome data with regard to readmission, and surgeon-specific post-operative management guidelines. While adoption of universal parameters for urinary catheter removal and post-operative mobilization have likely served to mitigate some of these issues, their impact remains uncertain in the current data set. In addition, the surgical scheduling of elective cases is a department-specific process rather than centralized with preservation of service line block time. Hence, the possibility of service line-specific bias in case-mix scheduling could be present within these data. Similarly, possible differences in weekend discharge resources were not considered in cases performed on Fridays, as day of the week effect analysis was not performed.

Overall, these data support the concept that late start (after 3 PM) elective surgical cases result in an increase in median LOS for patients, specifically those undergoing Orthopedic and Neurosurgical cases. From a cost perspective, this increase LOS by one day represents a total of 487 inpatient hospitalization days for which there is no increased revenue generation, and a decrease in occupancy capacity. In an era of emphasizing increased value and expenditure capitation, additional attention spent on optimizing the scheduling of elective surgical cases has the potential for improvement for both patients and healthcare delivery systems. To that end, a centralized approach to the scheduling of elective surgical cases and recognition of the need for cases to be started in a timely manner during daytime business hours may offer an improved method for decreasing unnecessary LOS increases over time.

\section{Conclusions}

Surgical scheduling of elective procedures with planned inpatient hospitalizations to start after 3 PM has the potential to lengthen stay. Such increased length of stay may result in opportunity loss for elective 
procedure margin, indicating a role for consideration of developing institutional strategies for scheduling optimization. In the post-COVID-19 era and with the emergence of value-based payments, both patient demand and institutional fiscal requirements stand to stress an already imperfect system. In reflecting on strategies for optimizing throughput, we believe that examination of surgical start time may provide a value benefit for patients and providers.

\section{Additional Information \\ Disclosures}

Human subjects: Consent was obtained or waived by all participants in this study. West Virginia University Institutional Review Board issued approval Exempt. Animal subjects: All authors have confirmed that this study did not involve animal subjects or tissue. Conflicts of interest: In compliance with the ICMJE uniform disclosure form, all authors declare the following: Payment/services info: All authors have declared that no financial support was received from any organization for the submitted work. Financial relationships: All authors have declared that they have no financial relationships at present or within the previous three years with any organizations that might have an interest in the submitted work. Other relationships: All authors have declared that there are no other relationships or activities that could appear to have influenced the submitted work.

\section{References}

1. Farley KX, Anastasio AT, Premkumar A, Boden SD, Gottschalk MB, Bradbury TL: The influence of modifiable, postoperative patient variables on the length of stay after total hip arthroplasty. J Arthroplasty. 2019, 34:901-6. 10.1016/j.arth.2018.12.041

2. Rodriguez JL, Brill JP, Hoth JJ, Stassen NA, Franklin GA, Barbier R, Polk HC: Tertiary care centers: a strategy for fiscal survival. Surgery. 2003, 134:684-9. 10.1016/S0039-6060(03)00329-5

3. Chin KR, Pencle FJ, Seale JA, Pencle FK: Clinical outcomes of outpatient cervical total disc replacement compared with outpatient anterior cervical discectomy and fusion. Spine. 2017, 42:E567-74. 10.1097/BRS.0000000000001936

4. Pasternack JB, Mahmood B, Martins AS, Choueka J: The transition of total elbow arthroplasty into the outpatient theater. JSES Int. 2020, 4:44-8. 10.1016/j.jses.2019.10.004

5. Chambers M, Huddleston JI, Halawi MJ: Total knee arthroplasty in ambulatory surgery centers: the new reality!. Arthroplast Today. 2020, 6:146-8. 10.1016/j.artd.2020.03.004

6. Healthcare Cost and Utilization Project (HCUP) Statistical Briefs . 2006. https://www.hcupus.ahrq.gov/reports/statbriefs/sb160.jsp.

7. Adamson T, Godil SS, Mehrlich M, Mendenhall S, Asher AL, McGirt MJ: Anterior cervical discectomy and fusion in the outpatient ambulatory surgery setting compared with the inpatient hospital setting: analysis of 1000 consecutive cases. J Neurosurg Spine. 2016, 24:878-84. 10.3171/2015.8.SPINE14284

8. Smith M II, Wheeler W, Ulmer MB: Comparison of outpatient laparoscopic cholecystectomy in a private nonteaching hospital versus a private teaching community hospital. JSLS. 1997, 1:51-3.

9. Edwards PK, Milles JL, Stambough JB, Barnes CL, Mears SC: Inpatient versus outpatient total knee arthroplasty. J Knee Surg. 2019, 32:730-5. 10.1055/s-0039-1683935

10. Mayo BC, Massel DH, Bohl DD, Long WW, Modi KD, Singh K: Effect of surgery start time on day of discharge in anterior cervical discectomy and fusion patients. Spine. 2016, 41:1939-44. 10.1097/BRS.0000000000001627

11. Feldhaus I, Mathauer I: Effects of mixed provider payment systems and aligned cost sharing practices on expenditure growth management, efficiency, and equity: a structured review of the literature. BMC Health Serv Res. 2018, 18:996. 10.1186/s12913-018-3779-1

12. Jubelt LE, Goldfeld KS, Blecker SB, et al.: Early lessons on bundled payment at an academic medical center . I Am Acad Orthop Surg. 2017, 25:654-63. 10.5435/JAAOS-D-16-00626

13. Wright MC, Phillips-Bute B, Mark JB, Stafford-Smith M, Grichnik KP, Andregg BC, Taekman JM: Time of day effects on the incidence of anesthetic adverse events. Qual Saf Health Care. 2006, 15:258-63. 10.1136/qshc. 2005.017566

14. O'Reilly-Shah VN, Melanson VG, Sullivan CL, Jabaley CS, Lynde GC: Lack of association between intraoperative handoff of care and postoperative complications: a retrospective observational study. BMC Anesthesiol. 2019, 19:182. 10.1186/s12871-019-0858-8

15. Guidry CA, Davies SW, Willis RN, Dietch ZC, Shah PM, Sawyer RG: Operative start time does not affect postoperative infection risk. Surg Infect. 2016, 17:547-51. 10.1089/sur.2015.150

16. Araujo RL, Karkar AM, Allen PJ, et al.: Timing of elective surgery as a perioperative outcome variable: analysis of pancreaticoduodenectomy. HPB. 2014, 16:250-62. 10.1111/hpb.12107

17. Kelz RR, Tran TT, Hosokawa P, et al.: Time-of-day effects on surgical outcomes in the private sector: a retrospective cohort study. J Am Coll Surg. 2009, 209:434-445.e2. 10.1016/j.jamcollsurg.2009.05.022

18. Lu Q, Li QS, Zhang W, et al.: Operation start time and long-term outcome of hepatocellular carcinoma after curative hepatic resection. Ann Surg Treat Res. 2020, 99:1-7. 10.4174/astr.2020.99.1.1

19. Addis DR, Moore BA, Garner CR, Fernando RJ, Kim SM, Russell GB: Case start time affects intraoperative transfusion rates in adult cardiac surgery: a single-center retrospective analysis. J Cardiothorac Vasc Anesth. 2020, 34:632-9. 10.1053/j.jvca.2019.10.044

20. Yount KW, Lau CL, Yarboro LT, Ghanta RK, Kron IL, Kern JA, Ailawadi G: Late operating room start times impact mortality and cost for nonemergent cardiac surgery. Ann Thorac Surg. 2015, 100:1653-8; discussion 1658-9. 10.1016/j.athoracsur.2015.04.131 